\title{
Xenografting for disease modeling of intramedullary spinal cord tumors: a systematic review
}

\author{
L. Rauschenbach ${ }^{1,2} \cdot$ E. Kocakavuk ${ }^{2} \cdot$ D. Pierscianek ${ }^{1} \cdot$ Y. Ahmadipour ${ }^{1} \cdot$ S. Kebir ${ }^{2,3} \cdot$ M. Glas $^{2,3} \cdot$ B. Scheffler ${ }^{2}$. \\ U. Sure ${ }^{1} \cdot$ R. Jabbarli ${ }^{1}$
}

Received: 29 November 2018 / Revised: 12 January 2019 / Accepted: 16 January 2019 / Published online: 1 February 2019

(C) International Spinal Cord Society 2019

\begin{abstract}
Study design Systematic review.

Objectives The overall incidence of intramedullary spinal cord tumors (IMSCT) remains low and clinical trials or standardized treatment strategies are missing. Therefore, multiple animal-based xenograft models (AXM) have been developed to foster preclinical research efforts on IMSCT. We constructed a systematic literature review to summarize and compare all AXM for IMSCT, published until April 16, 2018.

Methods The review was conducted using 4 independent research databases following the PRISMA (preferred reporting items for systematic reviews and meta-analyses) guidelines. Studies were included, if they reported on surgical transplantation of tumor cells or tumor tissue to the spinal cord. Methodological study quality was assessed according to the SYRCLE (systematic review center for laboratory animal experimentation) risk of Bias tool.

Results Systematic search yielded 20 publications dealing with AXM for IMSCT. In summary, 4 tumor entities were analyzed in 23 experiments using 337 animals, mainly investigating glioblastoma or gliosarcoma biology. Studies varied regarding the use of engrafted animals, surgical techniques and tumor burden. Most commonly authors used heterotopic, transdural injection of immortalized brain tumor cell lines $\left(1 \times 10^{5}\right.$ in $\left.5 \mu \mathrm{l}\right)$ into the thoracic spinal cord of immunocompromised rats. Quality assessment demonstrated an unclear risk of bias in most cases.

Conclusion Although different AXM for IMSCT have been described so far, one rat model is technically feasible, enables robust experiments and demonstrates reproducible results. However, there is a need for new AXM using orthotopic engraftment of patient-derived tumor cells and for genetically engineered animal models.
\end{abstract}

Supplementary information The online version of this article (https:// doi.org/10.1038/s41393-019-0248-6) contains supplementary material, which is available to authorized users.

$\triangle$ L. Rauschenbach

laurel.rauschenbach@uk-essen.de

1 Department of Neurosurgery, University Hospital Essen, Essen, Germany

2 DKFZ Division of Translational Neurooncology at the West German Cancer Center (WTZ), German Cancer Consortium (DKTK) Partner Site, University Hospital Essen, Essen, Germany

3 Division of Clinical Neurooncology, Department of Neurology, University Hospital Essen, Essen, Germany

\section{Introduction}

Intramedullary spinal cord tumors (IMSCT) comprise a heterogeneous group of benign and malignant tumor entities and account for $2-4 \%$ of all central nervous system tumors, with ependymoma and astrocytoma being the most common tumor entities in adult, adolescent and pediatric populations $[1,2]$. Less frequent tumor entities comprise hemangioblastomas, gangliogliomas, germinomas, primary central nervous system lymphomas or metastases from extraspinal tumor diseases. Although IMSCT are rare disorders compared to intracerebral tumors, there is a continuing increase in clinical incidences and thus growing need for treatment options [3]. While most IMSCT are classified as benign tumors, several tumor entities can exhibit malignant properties with local infiltration of spinal cord parenchyma and adjacent structures, resulting in diffuse or radicular back pain, 
sensory changes, motor deficits or bowel, and bladder dysfunctions [4]. Although IMSCT can be located throughout the whole spinal cord, they usually grow in the cervical spinal cord, with less frequent tumor growth in the thoracic and lumbar spinal cord [5].

Since neurooncologic research mainly focuses on intracerebral tumors, scientific knowledge of spinal tumor biology is still in its infancy [6]. Considering the relatively small population size of people suffering from IMSCT, there is an unmet need for clinical trials with a larger sample size investigating new anticancer strategies and evidence-based treatment procedures. Currently, these tumors are treated with neurosurgical resection and sometimes additionally with radiation and chemotherapy in case of malignancy or tumor recurrence [7]. However, unlike extramedullary tumors, gross total resection is often not feasible and patients only receive tumor debulking, frequently resulting in recurrent tumor growth with tremendous functional impairment, discouraging clinical deterioration and premature death $[8,9]$. In these cases, postoperative adjuvant therapy has a pivotal role.

To investigate for effective radio-therapeutic and chemotherapeutic treatment approaches, preclinical animal models which reflect clinically relevant features of tumor biology are needed. In the last decades, numerous animalbased xenograft models (AXM) have been developed to mimic human tumor growth in non-human organisms [10]. Notably, AXM are widely accepted tools in all fields of oncological research, since they have fundamentally contributed to the understanding of pathophysiological mechanisms and to the development of novel therapies. Although translational significance is sometimes limited, these models are indispensably needed to test preclinical results for their clinical applicability. In summary, general agreement exists that a satisfying AXM should meet a setup of different criteria: The AXM should be (a) reproducible, (b) technically feasible, (c) cost-effective for large scale testing, (d) comparable with human disease with enough survival time for therapeutic interventions and finally (e) amenable to non-invasive disease monitoring [11].

So far, several AXM have been described, however investigators have used different organisms, different transplantation techniques and different tumor types. This is the first review which aims to summarize all AXM for IMSCT published until April 2018. With a special focus on technical advantages, effectiveness and feasibility we compare these models in order to determine limitations and future challenges. In the long-term perspective, a widely accepted animal model remains to be determined that is amenable to the testing of promising therapeutics for new treatment paradigms in clinical care.

\section{Methods}

\section{Search strategy}

The systematic review of literature was performed in accordance with PRISMA (preferred reporting item for systematic reviews and meta-analyses) guidelines (see Fig. 1). All searches were conducted on April 16, 2018. In order to provide a comprehensive review of all reported AXM for neoplastic spine diseases, we used a broad search strategy. The authors performed a computerized literature search using PubMed/MEDLINE, Web of Science, Scopus and Cochrane Library to exclude database bias. A summary of search strings is provided (see Supplemental Content, Table 1). Manual search with other search strings was run to detect suitable articles not identified in the database searches. Additionally, authors were asked to contribute eligible articles that were not found by other searching means. All duplicates were excluded. Titles and abstracts of remaining publications were reviewed and suitable articles were extracted. The references of retrieved publications were reviewed and additional citations were extracted. Research studies were finally eligible for inclusion if they addressed AXM for IMSCT, published in English since 1950. Conference papers, reviews, case reports, notes and book chapters were excluded. The resulting publication list was used to prepare this review and all included studies were subsequently analyzed in detail.

\section{Data collection}

All articles were independently screened by two investigators for their suitability (LR, EK). A third investigator decided in cases of disagreement (RJ). The final publication list was analyzed by two investigators (LR, EK) and relevant data points were extracted with special attention to surgical engraftment techniques (engrafted tumor entity, engrafted tumor material, mode of transplantation, number of tumor cells, amount of tumor tissue, volume of liquid medium, surgical approach), effectiveness (incidence of surgery related death, relative number of symptomatic animals, symptoms of successfully engrafted organisms, overall survival of successfully engrafted organisms) and feasibility (recipient organism, number of engrafted organisms, time from engraftment to symptomatic manifestation). Information on outcome were identified from both texts and graphs. When the number of animals was reported as a range, the lowest group size was collected. We assumed no exclusion of animals, when the number of animals in the method section was equal to the number of animals in the results section. Orthotopic engraftment was assumed if IMSCT were transplanted into the spinal cord of recipient organisms; all other engraftment approaches were defined 


\section{Identification}

Identifying records through database search

$(\mathrm{n}=3.562)$ :

- PubMed/MEDLINE ( $n=643)$

- Scopus $(n=1.192)$,

- Web of Science $(n=1.689)$

- Cochrane $(n=38)$

Removing duplicates $(n=1.374)$

Identifying additional records through manual search / author's knowledge $(n=0)$

Included records: $n=2.188$

\section{Screening}

Screening of title and abstract $(n=2.188)$ and exclusion of records that meet exclusion criteria

$(n=2.037)$. Exclusion criteria:

- Study published prior 1950.

- Review, conference paper, book chapter, case report, note.

- Study only with abstract.

- Study without English translation.

- In vitro study.

- In vivo study performed in humans.

- In vivo study without engraftment.

- In vivo study investigating non-tumor diseases.

- In vivo study investigating intracerebral tumor diseases.

- In vivo study investigating leptomeningeal tumor dissemination to the spine.

- In vivo study investigating metastatic tumor diseases without spine involvement.

Screening of references and inclusion of records that do not meet exclusion criteria $(n=38)$.

Included records: $n=187$

\section{Eligibility and inclusion}

Full-text review $(n=187)$ and exclusion of records that meet exclusion criteria $(n=12)$. Exclusion of nonintramedullary tumors $(n=155)$. Final inclusion of studies $(n=20)$

Fig. 1 Systematic literature search. Finally, 20 studies dealing with AXM for IMSCT were included

as heterotopic. Data that was mentioned but not shown was not included into this review.

\section{Quality assessment}

Methodological quality of all studies was assessed according to the SYRCLE (systematic review center for laboratory animal experimentation) risk of bias tool for animal studies
[12]. Two authors independently investigated all studies for all 10 SYRCLE items (LR, EK). A third author decided in cases of disagreement (RJ). We additionally investigated all the studies for the following items in order to improve quality assessment: (item 11) mention of randomization at any level, (item 12) detailed description of the surgical procedure, (item 13) detailed elaboration of surgical safety of the procedure, (item 14) detailed information on the translational significance.

\section{Role of funding source}

This study has no funding source. The authors LR, EK, US, and RJ had full access to all data in this study. LR and RJ took the final responsibility for the decision to submit this manuscript.

\section{Results}

\section{Study selection}

We performed a systematic literature review and based on inclusion criteria, we identified a total of 175 studies which reported on AXM experiments for spinal tumors (see Fig. 1). Only 20 studies referred to IMSCT and were finally included. Additional search with other search strings did not yield further records. According to the authors' expertize and to the best of their knowledge they could not identify additional relevant articles.

\section{Study characteristics}

\section{Organisms and engrafted material}

We reviewed 20 studies dealing with 23 experiments and comprising a total of approximately 337 animals with a median of 12 animals per study (see Table 1). Glioblastoma WHO $\mathrm{IV}^{\circ}$ and Gliosarcoma $\mathrm{WHO} \mathrm{IV}^{\circ}$ were used in most experiments $(91 \%, n=21)$, one study investigated for atypical teratoid / rhabdoid tumors $\mathrm{WHO} \mathrm{IV}^{\circ}(4 \%, n=1)$ and one study used unspecified squamous cell carcinoma (4\%, $n=1)$. Most of the studies engrafted tumor cells $(96 \%, n=$ $22)$, only one used tumor tissue $(4 \%, n=1)$. As a recipient animal species, most researchers used immunocompromised rats $(87 \%, n=20)$, other experiments were conducted with mice $(4 \%, n=1)$, rabbits $(4 \%, n=1)$ or dogs $(4 \%, n=1)$. Gender of the recipient organisms was often not mentioned, but choices for male $(30 \%, n=7)$ or female $(35 \%, n=8)$ animals were balanced and consistent in each experiment. In 23 transplantation experiments, authors usually used commercially available and immortalized chemical-induced brain tumor cell lines $(57 \%, n=13)$ or human tumor cell 


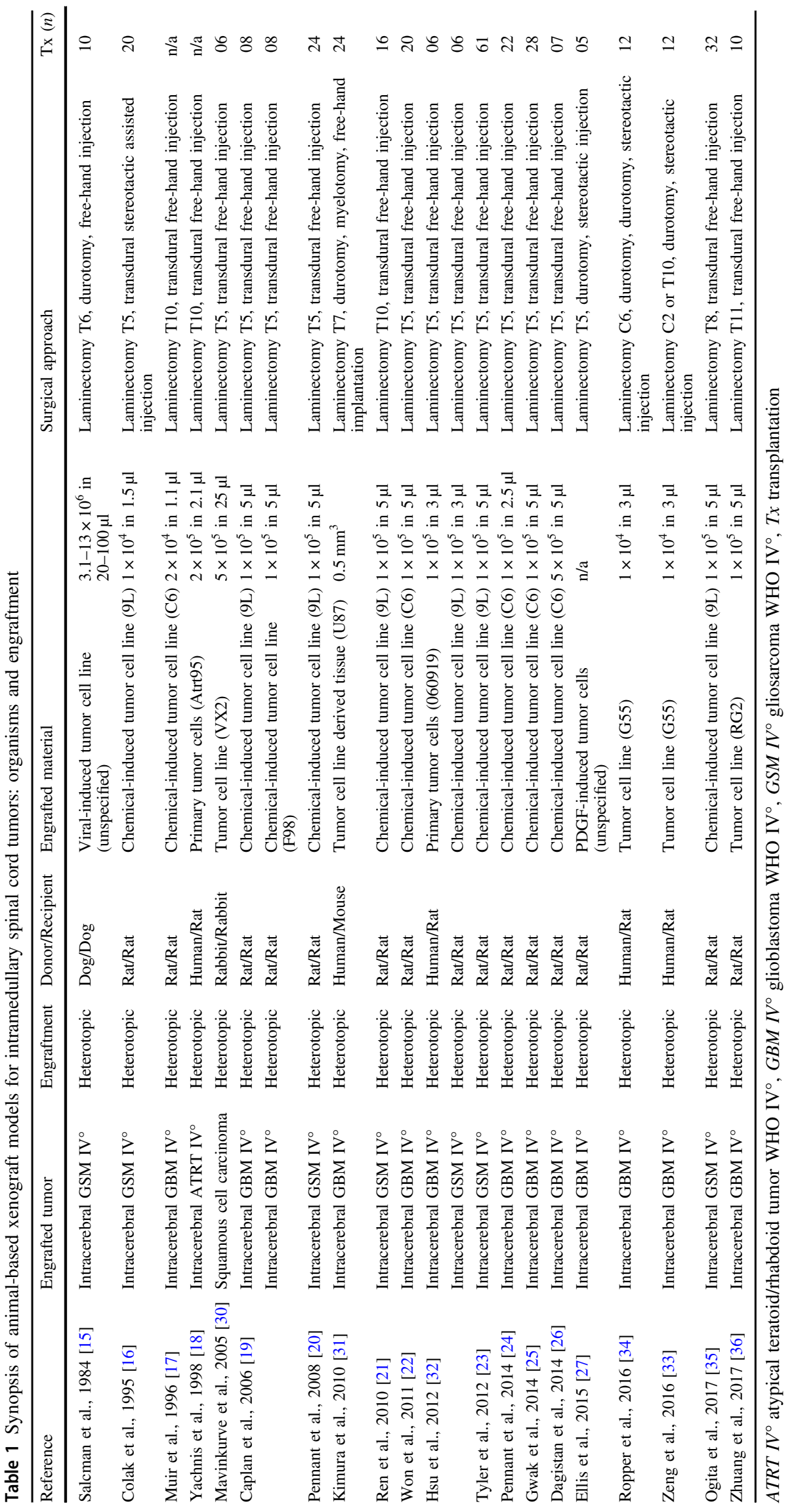


lines $(22 \%, n=5)$ for tumor engraftment. Patient-derived tumor cells $(9 \%, n=2)$, rodent tumor cell lines $(4 \%, n=1)$, viral-induced tumor cells $(4 \%, n=1)$ and gene-induced tumor cells $(4 \%, n=1)$ were used less frequently. Engraftment was exclusively restricted to heterotopic tumor transplantation $(100 \%, n=23)$ but there was no study that used orthotopic transplantation of tumor cells originating from IMSCT. Tumor burden varied between these studies, dependent on the recipient organism and on the tumor composition. Median injection into the spinal cord of rats consisted of $1 \times 10^{5}$ tumor cells (range: $1 \times 10^{4}-5 \times 10^{5}$ ) in $5 \mu \mathrm{l}$ medium (range: $1.1-5 \mu \mathrm{l}$ ). One research group investigated tumor tissue and engrafted a tissue cube of $0.5 \mathrm{~mm}^{3}$ into mice.

In summary, heterotopic transplantation of $1 \times 10^{5}$ immortalized and chemical-induced brain tumor cell lines into the spinal cord of immunocompromised rats was the most commonly used AXM across all experiments $(52 \%, n=12)$.

\section{Surgical approach}

All experiments were reviewed for their mode of tumor engraftment. Notably, all authors used a posterior surgical approach in their experiments, performing laminectomy of one or two segments in the cervical $(9 \%, n=2)$ or thoracic (91\%, $n=21$ ) dorsal column (Tab. 1). In most cases, an incision was made in the dorsal mid-thoracic region to expose and remove the spinous process of T5, T6, T7 or T8 with rongeurs $(76 \%, n=16)$. However, few authors used a caudal-thoracic approach and removed T10 or T11 $(22 \%$, $n=5$ ). We identified only two studies where the cervical dorsal column was accessed with a laminectomy of $\mathrm{C} 2$ or C6 $(9 \%, n=2)$. In most cases, the ligamentum flavum was removed and tumor cell suspension was injected intramedullary through the dura mater with a syringe $(74 \%, n=$ 17). However, if tumor tissue was implanted or if authors feared syringe dislocation, durotomy was performed $(26 \%$, $n=6)$. In contrast to AXM for intracerebral tumors, injections were usually not assisted stereotactically $(17 \%, n=4)$ but performed by free-hand $(83 \%, n=19)$. In case of stereotactically assisted tumor cell injection, all authors defined the posterior median sulcus as a target structure (0.5-2 $\mathrm{mm}$ beneath the dorsal pial surface).

\section{Applicability}

While most experiments developed new AXM for IMSCT $(57 \%, n=13)$, others tested treatment approaches $(39 \%$, $n=9$ ) or investigated tumor biology $(4 \%, n=1)$ (see Table 2). Although incidence of surgery-related death was often not specified, no author reported lethal complications. Consistent with human disease, most experiments used motor deficits as an outcome parameter $(91 \%, n=21)$. Since Basso, Beattie and Bresnahan have created a locomotor rating scale in 1995 , i.e., BBB score, this tool was routinely used in many experiments to describe locomotor deficits in rats $(67 \%, n=14)$. In all cases where animals were clinically monitored, hind limb paresis occurred inevitably after few days (mean BBB score day 5: $16 \pm 2$; consistent plantar stepping and consistent forelimbhindlimb coordination during gait; and toe clearance occurs frequently during forward limb advancement; predominant paw position is parallel at initial contact and rotated at lift off) and deteriorated to paraplegia a few days later (mean decrease of BBB score between day 5 and 10: 7 \pm 3 ; occasional weight supported plantar steps, no forelimb forelimb-hindlimb coordination). Moreover, xenotransplantated animals often suffered from weight loss, abnormal behavior or posturing, which is a term often used to describe kyphosis or hunchback. If cervical spinal cord was used for engraftment, animals regularly suffered from autonomic dysfunctions, namely from blood pressure and body temperature changes $(50 \%, n=1)$. In most cases paraplegia was defined as an outcome parameter and in accordance with ethical committees animals were sacrificed at this stage of disease, resulting in median survival rates of 17 days (11-33 days) for experiments with rats. Apparently, animals bearing patient-derived tumor cells revealed longest overall survival, compared to animals engrafted with commercially available cell lines. To validate tumor growth or to monitor tumor volume changes in vivo, several studies used radiographic tools $(39 \%, n=9)$, namely magnetic resonance imaging $(78 \%, n=7)$, bioluminescence imaging (11\%, $n=1)$ or myelography together with computed tomography imaging $(11 \%, n=1)$. Finally, tumor growth was verified in almost every experiment post mortem $(96 \%$, $n=22$ ) via necropsy.

\section{Quality assessment and risk of bias}

The quality assessment according to the SYRCLE risk of bias tool demonstrates poor reporting, resulting in an unclear risk of bias in most cases (see Fig. 2). While many studies revealed similarity at animal baseline (item 2 "yes": $n=9,45 \%$ ) and reported on data outcome (item 8 "yes": $n=12$, 60\%) no author commented on randomization procedures (item 1 "yes": $n=0,0 \%$; item 3 "yes": $n=0$, $0 \%$ ). Data to assess the risk of performance bias (item 4 "yes": $n=0,0 \%$; item 5 "yes": $n=1,5 \%$ ), detection bias (item 6 "yes": $n=0,0 \%$; item 7 "yes": $n=0,0 \%$ ) and reporting bias (item 9 "yes": $n=1,5 \%$ ) were incomplete, leading to an unclear risk of bias in most studies. Regarding the poor reporting on SYRCLE items, we additionally created a portfolio of 4 items for this review as described in the method section. Randomization at any level was 


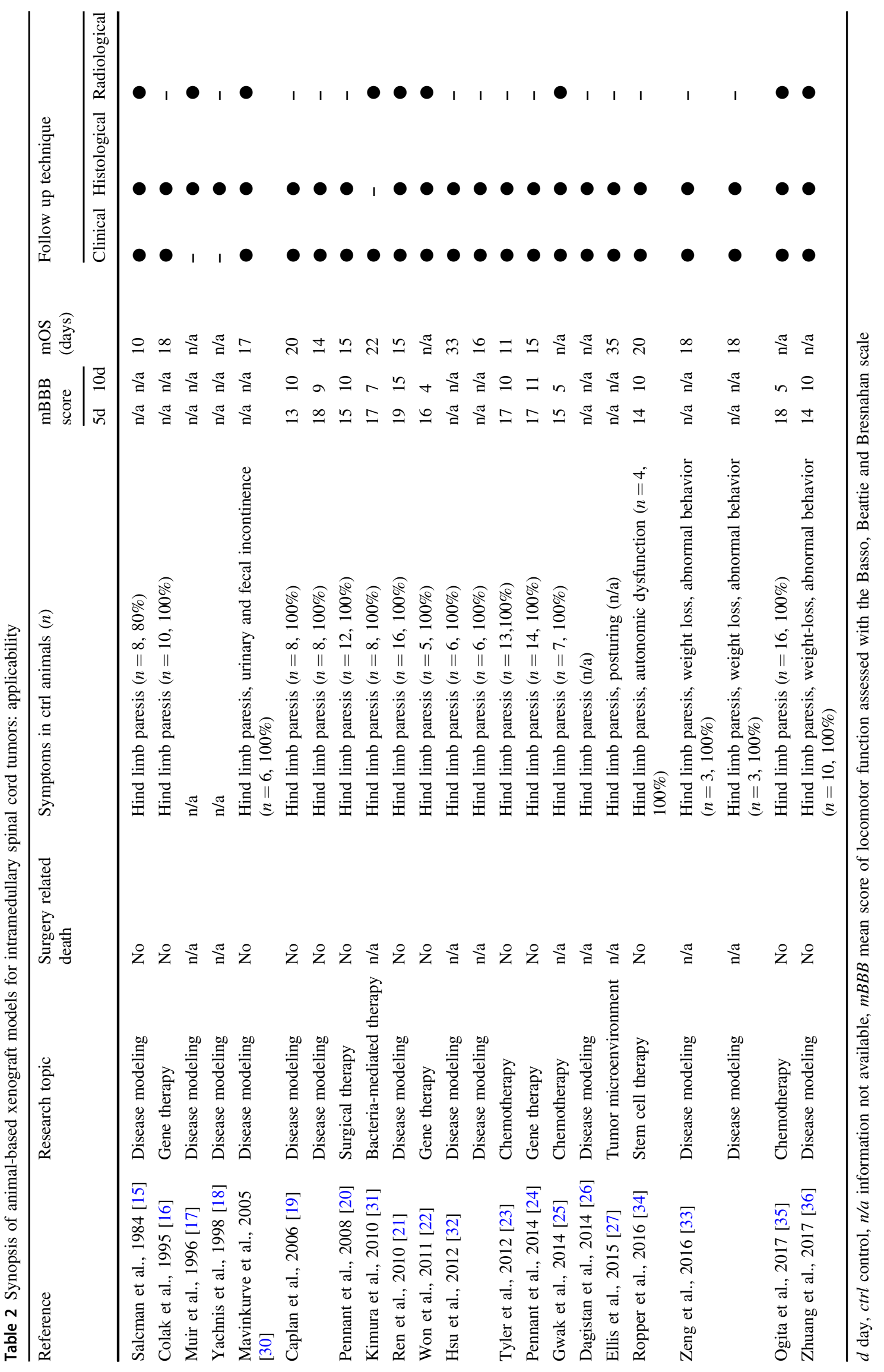




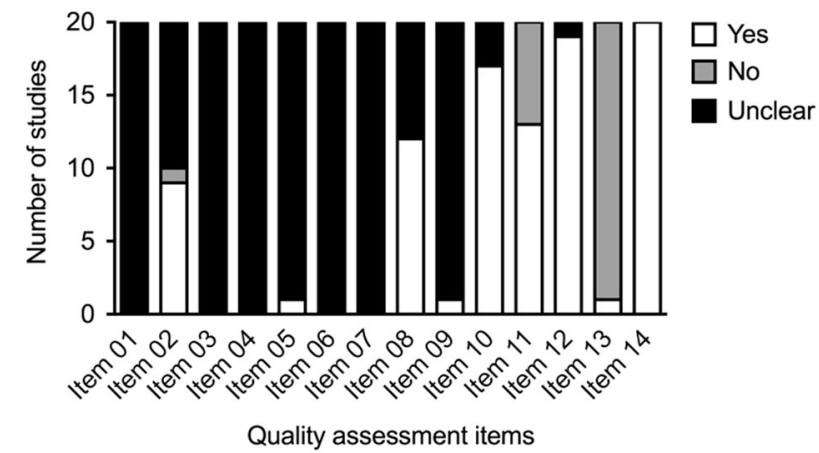

Fig. 2 Quality assessment according to the SYRCLE Risk of Bias tool. The number of studies $(n=20)$ meeting each quality score criterion $(n=14)$ is illustrated. "Yes" means that the description of measurements meets the item requirements (low risk of bias). "No" means that the description does not meet the item requirements (high risk of bias). "Unclear" means there is no relevant information (unclear risk of bias). Items 11-14 were additionally created to improve quality assessment. Items: Item 01-Was the allocation sequence adequately generated and applied? Item 02-Were the groups similar at baseline or were they adjusted for confounders in the analysis? Item 03-Was the allocation of the different groups adequately concealed? Item 04-Were the animals randomly housed during the experiment? Item 05-Were the caregivers/investigators blinded from knowledge which intervention each animal received during the experiment? Item 06-Were the animals selected at random for outcome assessment? Item 07-Was the outcome assessor blinded? Item 08-Were incomplete outcome data adequately addressed? Item 09-Are reports of the study free of selective outcome reporting? Item 10- Was the study apparently free of other problems that could result in high risk of bias? Item 11-Was randomization mentioned at any level? Item 12-Was the surgical procedure described adequately? Item 13-Was the surgical safety mentioned? Item 14-Was translational significance elaborated?

reported frequently (item 11 "yes": $n=13,65 \%$ ) and surgical procedures were described adequately in almost every case (item 12 "yes": $n=19,95 \%$ ). However, information on surgery-related death was not mentioned (item 13 "yes": $n=1,5 \%$ ) but translational approach was always elaborated (item 14 "yes": $n=20,100 \%$ ).

\section{Discussion}

Care for patients who suffer from IMSCT is challenging due to their limited treatment options [13, 14]. Surgical resection will remain technically challenging and adjuvant treatment approaches are urgently needed to improve patient care. Nevertheless, efficacy of these treatment regimens remains controversial and only limited evidencebased data exists for clinical scenarios with high-grade IMSCT [7]. At the moment, no standardized and evidencebased chemotherapeutic regimens exist for most tumor entities that are treated beyond the context of clinical trials and optimal treatment has yet to be determined. Accordingly, clinical management of patients continues to be an unmet healthcare demand and promising research efforts are needed. However, the low incidence of IMSCT has hampered the study of these tumors and clinical trials with a large sample size are missing. Since progress in this field proceeds slowly, the National Institutes of Health Office of Rare Diseases Research, the National Cancer Institute of Neurological Disease and Stroke published a conference paper on future directions in spinal cord tumor research in 2010 [6]. In this publication, clinical and laboratory experts highlighted the urgent need for multicentric patient registration and tissue sampling, prospective clinical trials with large sample sizes, and intensified research efforts in the field of tumorigenesis and animal- based modeling. In an effort to test the efficacy and safety of novel therapeutics for IMSCT, researchers have developed a setup of reliable, predictive and reproducible AXM, beginning in 1984 by Salcman and colleagues [15]. While this first study of this period was hardly reproducible and lacked feasibility due to the usage of dogs, later studies could overcome these limitations with the creation of small animal approaches.

Using the database search procedures outlined above, we have to the best of our knowledge reviewed all relevant studies dealing with AXM for IMSCT, with special emphasis on technical procedures, effectiveness and feasibility.

In conclusion and based upon comparison between all AXM for IMSCT, we are convinced that intramedullary transplantation of tumor cells into the spinal cord of immunocompromised rats is currently the most established and most suitable disease model. Numerous studies have demonstrated that surgical intervention on the spinal cord of rats is safe and feasible [16-27]. Furthermore, housing of these organisms is easy and cost effective [28]. All studies we reviewed demonstrated fast tumor growth within few weeks and clinical deterioration in all animals, allowing non-invasive disease-monitoring and experimental intervention at the time of disease onset. Locomotor function can easily be determined and scored according to the wellestablished and validated rating scale for rats with contusion spinal cord trauma, described by Basso and colleagues [29]. Nevertheless, most of these AXM are immunocompromised and the effect of the immune system on tumor growth has to be considered carefully in these models.

Systematic literature review revealed different transplantation techniques. Surgical approach to the spinal cord was always performed using a laminectomy of one or two spinal column segments. In most cases, tumor cells and not tumor tissue were injected in the mid-thoracic spinal cord, which routinely led to hind limb paresis and finally to paraplegia $[15,16,19,20,22-27,30-33]$. Although cervical spinal cord might be more suitable for tumor cell transplantation regarding the increased volume of central nervous tissue, this approach was less frequently performed and routinely caused autonomic dysfunction $[33,34]$. The 
majority of authors preferred transdural injection of tumor cells into the spinal cord and this technique has to be considered as being safe [16-26, 30, 32, 35, 36]. However, if possible, injection should be stereotactically supported in order to improve the trajectory of the syringe and to target the depth of the posterior median sulcus [16, 27, 33, 34].

So far, only 2 studies have used patient-derived tumor cells while most authors performed transplantation of commercially available and immortalized cell lines [18, 32]. Hsu and colleagues engrafted patient-derived glioblastoma neurospheres into the spinal cord of immunocompromised rats and demonstrated tumor growth in all animals [32]. Also, transplanted tumor cells captured the growth pattern and local invasive nature of their human counterparts and animals demonstrated prolonged survival times compared to other models, indicating a more realistic AXM for IMSCT. Although tumor cell lines have been used for decades in all fields of oncological research, growing evidence exists that patient-derived tumor cells might be more suitable for disease modeling, since they recapitulate tumor heterogeneity and reveal individual therapeutic resistance, giving a more representative model of human malignancies [37, 38]. In this regard, also the unique tumor environment of the spinal cord must be considered. Ellis and colleagues transplanted retroviral-mediated and platelet-derived growth factor (PDGF) induced glioblastoma cells into the forebrain and into the spinal cord of immunocompromised rats and demonstrated that tumor phenotypes were consistently predicted by the tissue into which tumor cells were transplanted rather that by the tissue of origin [27]. Thus, microenvironment of the spinal cord seems to be unique and might be different from the brain. Regarding this observation one may hypothesize that orthotopic engraftment of IMSCT into the spinal cord is more representative than the use of heterotopically transplanted intracerebral tumors. However, more laboratory studies are needed to confirm this hypothesis.

Quality assessment in this review revealed insufficient reporting on methods and results in most studies, resulting in an overall unclear risk of bias. Interestingly, a trend towards better reporting quality in more recent studies could not be detected. The SYRCLE risk of bias tool is a recently published quality assessment method to improve critical appraisal of evidence from animal studies and is adapted from the Cochrane risk of bias tool [12]. Nevertheless, the lack of detailed reporting is a well-known obstacle in animal studies and critical evaluation of bias, e.g., randomization and blinding, is not yet established in this field of research so far [39]. In accordance with the SYRCLE group, we did not use a scoring system to quantify reporting quality of each individual study, because overall reporting was low and systematic evaluation of animal-based studies is not yet established [12]. Nevertheless, since we found that the risk of bias could not be estimated in most experiments, methodological reporting has to improve. While surgical procedures and translational significance were often elaborated appropriately, many studies lacked detailed reporting on animal randomization, blinding of investigators and completeness of outcome data.

This report gives an overview on all available AXM for IMSCT that have been published so far and that can be used to extend in vitro data to in vivo experiments. In line with our review, transdural injection of tumor cell lines $\left(1 \times 10^{5}\right.$ in $5 \mu \mathrm{l}$ ) into the thoracic spinal cord of immunocompromised rats might be considered safe, reproducible and the most extensively validated model for IMSCT.

Although low-grade ependymomas and astrocytomas are the most common tumor entities in the spinal cord, all studies we have identified were limited to high-grade glioblastoma, gliosarcoma or rare tumor entities. Thus, we strongly recommend to expand the field of research on other IMSCT with a higher clinical incidence, using the abovementioned animal model. However, further studies are needed to develop more suitable AXM, using patientderived tumor cells and experimental setups with less risk of bias. Furthermore, transgenic animal models are warranted, especially for low-grade tumor entities, that often do not develop sufficient in vivo tumor growth after cell engraftment [40].

\section{Conclusion}

Despite growing research efforts, malignant IMSCT inflict considerable morbidity and mortality given their limited treatment options and their high recurrence rate. Future studies should investigate new therapeutic strategies in orthotopic and immunocompromised rat xenograft models using patient-derived tumor cells. Additionally, research should also focus on tumorigenesis in human IMSCT to establish robust genetically engineered animal models.

\section{Data archiving}

The dataset generated during the current study is available in the PubMed/MEDLINE, Scopus, Web of Science and Cochrane Library databases:

https://www.ncbi.nlm.nih.gov/pubmed/

https://www.scopus.com/

https://login.webofknowledge.com/

https://www.cochranelibrary.com/

Detailed data summaries are available from the corresponding author on reasonable request. 
Acknowledgements We thank Leonie Debes (Faculty of Veterinary Medicine, University of Leipzig / Germany) for her helpful advice on various veterinarian issues examined in this publication.

Author contributions: LR was responsible for designing the review protocol, writing the protocol and report, conducting the search, screening potentially eligible studies, extracting and analyzing data, interpreting results, updating the reference list and creating figures and tables. EK was responsible for designing the review protocol, screening potentially eligible studies, extracting and analyzing data and providing feedback on the report. DP was responsible for interpreting results and providing feedback on the report. YA was responsible for interpreting results and providing feedback on the report. SK was responsible for interpreting results and providing feedback on the report. MG was responsible for interpreting results and providing feedback on the report. BS was responsible for interpreting results and providing feedback on the report. US was responsible for providing feedback on the report. RJ was responsible for designing the review protocol, writing the protocol and report, conducting the search, screening potentially eligible studies, extracting and analyzing data, interpreting results and providing feedback on the report.

\section{Compliance with ethical standards}

Conflict of interest The authors declare that they have no conflict of interest.

Ethical statement This article does not contain any studies involving animals or human participants performed by any of the authors.

Publisher's note: Springer Nature remains neutral with regard to jurisdictional claims in published maps and institutional affiliations.

\section{References}

1. Chamberlain MC, Tredway TL. Adult primary intradural spinal cord tumors: a review. Curr Neurol Neurosci Rep. 2011;11:320-8.

2. Adams H, Avendano J, Raza SM, Gokaslan ZL, Jallo GI, Quinones-Hinojosa A. Prognostic factors and survival in primary malignant astrocytomas of the spinal cord: a population-based analysis from 1973 to 2007. Spine. 2012;37:727-35.

3. Hsu S, Quattrone M, Ostrom Q, Ryken TC, Sloan AE, BernholtzSloan JS. Incidence patterns for primary malignant spinal cord gliomas: a surveillance, epidemiology, and end results (SEER) study. J Neurosurg Spine. 2011;14:742-7.

4. Raco A, Esposito V, Lenzi J, Piccirilli M, Delfini R, Cantore G. Long-term follow-up of intramedullary spinal cord tumors: a series of 202 cases. Neurosurgery. 2005;56:972-81.

5. Kane PJ, El-Mahdy W, Singh A, Powell MP, Crockard HA. Spinal intradural tumors: Part II - Intramedullary. Br J Neurosurg. 1999;13:558-63.

6. Claus EB, Abdel-Wahab M, Burger PC, Engelhard HH, Ellison DW. Defining future directions in spinal cord tumor research. J Neurosurg Spine. 2010;12:117-21.

7. Tobin MK, Geraghty JR, Engelhard HH, Linninger AA, Mehta AI. Intramedullary spinal cord tumors: a review of current and future treatment strategies. Neurosurg Focus. 2015;39:1-10.

8. Matsumoto T, Urasaki E, Soejima Y, Nakano Y, Yokota A, Nishizawa S. Cervical intramedullary glioblastoma: report of a long-term survival case and a review of the literature. J UOEH. 2008;30:413-20.
9. Samartzis D, Gillis CC, Shih P, O'Toole JE, Fessler RG. Intramedullary spinal cord tumors: Part I-Epidemiology, pathophysiology, and diagnosis. Global Spine J. 2015;5:425-35.

10. Jung J. Human tumor xenograft models for preclinical assessment of anticancer drug development. Toxicol Res. 2014;30:1-5.

11. Fomchenko El, Holland EC. Mouse models of brain tumors and their applications in preclinical trials. Clin Cancer Res. 2006;12:5288-97.

12. Hooijmans CR, Rovers MM, de Vries RB, Leenaars M, RitskesHoitinga M, Langendam MW. SYRCLE's risk of bias tool for animal studies. BMC Med Res Methodol. 2014;14:43. https://doi. org/10.1186/1471-2288-14-43.

13. Constantini S, Miller DC, Allen JC, Rorke LB, Freed D, Epstein FJ. Radical excision of intramedullary spinal cord tumors: surgical morbidity and long-term follow-up evaluation in 164 children and young adults. J Neurosurg. 2000;93:183-93.

14. Sandler HM, Papadopoulos SM, Thornton AF Jr, Ross DA. Spinal cord astrocytomas: results of therapy. Neurosurgery. 1992;30:490-3.

15. Salcman M, Botero E, Rao KC, Broadwell RD, Scott E. Intramedullary canine spinal cord tumor model. J Neurosurg. 1984;61:761-6.

16. Colak A, Goodman JC, Chen SH, Woo SL, Grossman RG, Shine HD. Adenovirus-mediated gene therapy for experimental spinal cord tumors: tumoricidal efficacy and functional outcome. Brain Res. 1995;691:76-82.

17. Muir D, Johnson J, Rojiani M, Inglis BA, Rojiani A, Maria BL. Assessment of laminin-mediated glioma invasion in vitro and by glioma tumors engrafted within rat spinal cord. J Neurooncol. 1996;30:199-211.

18. Yachnis AT, Neubauer D, Muir D. Characterization of a primary central nervous system atypical teratoid/rhabdoid tumor and derivative cell line: immunophenotype and neoplastic properties. J Neuropathol Exp Neurol. 1998;57:961-71.

19. Caplan J, Pradilla G, Hdeib A, Tyler BM, Legnani FG, Bagley CA. A novel model of intramedullary spinal cord tumors in rats: functional progression and histopathological characterization. Neurosurgery. 2006;59:193-200.

20. Pennant WA, Sciubba DM, Noggle JC, Tyler BM, Tamargo RJ, Jallo Gl. Microsurgical removal of intramedullary spinal cord gliomas in a rat spinal cord decreases onset to paresis, an animal model for intramedullary tumor treatment. Childs Nerv Syst. 2008;24:901-7.

21. Ren T, Wang ZC, Zhang YZ, Li D, Wang HY, Li ZZ. Establishment of intramedullary spinal cord glioma model in rats. Chin Med J. 2010;123:2580-5.

22. Won YW, Kim KM, An SS, Lee M, Ha Y, Kim YH. Suicide gene therapy using reducible poly (oligo-D-arginine) for the treatment of spinal cord tumors. Biomaterials. 2011;32:9766-75.

23. Tyler BM, Hdeib A, Caplan J, Legnani FG, Fowers KD, Brem H, et al. Delayed onset of paresis in rats with experimental intramedullary spinal cord gliosarcoma following intratumoral administration of the paclitaxel delivery system OncoGel. J Neurosurg Spine. 2012;16:93-101.

24. Pennant WA, An A, Gwak SJ, Choi S, Banh DT. Local non-viral gene delivery of apoptin delays the onset of paresis in an experimental model of intramedullary spinal cord tumor. Spinal Cord. 2014;52:3-8.

25. Gwak SJ, An SS, Yang MS, Joe E, Kim DH, Yoon DH, et al. Effect of combined bevacizumab and temozolomide treatment on intramedullary spinal cord tumor. Spine. 2014;39:65-73.

26. Dagistan Y, Ozyalvacli G, Firat T, Yagmurlu K, Terzi EH. Intramedullary spinal cord glioma following microinjection of glioblastoma cell line C6 in rats. Open J Mod Neurosurg. 2014;4:43-46. 
27. Ellis JA, Castelli M, Assanah M, Bruce JN, Canoll P, Ogden AT. Unique microenvironment responses to PDGF stimulation in brain and spinal cord gliomas determine tumor phenotype. J Neurooncol. 2015;123:27-33.

28. Basso DM, Beattie MS, Bresnahan JC. A sensitive and reliable locomotor rating scale for open field testing in rats. J Neurotrauma. 1995;12:1-21.

29. John BA, Said N. Insights from animal models of bladder cancer: recent advances, challenges, and opportunities. Oncotarget. 2017;8:57766-81.

30. Mavinkurve G, Pradilla G, Legnani FG, Tyler BM, Bagley CA, Brem $\mathrm{H}$, et al. A novel intramedullary spinal cord tumor model: functional, radiological, and histopathological characterization. J Neurosurg Spine. 2005;3:142-8.

31. Kimura $\mathrm{H}$, Zhang L, Zhao M, Hayashi $\mathrm{K}$, Tsuchiya $\mathrm{H}$, Tomita K, et al. Targeted therapy of spinal cord glioma with genetically modified salmonella typhimurium. Cell Prolif. 2010; 43:41-48

32. Hsu W, Siu IM, Paradilla G, Gokaslan ZL, Jallo GI, Gallia GL. Animal model of intramedullary spinal cord glioma using human glioblastoma multiforme neurospheres. J Neurosurg Spine. 2012;16:315-9.

33. Zeng X, Han I, Abd-El-Barr M, Aljuboori Z, Anderson JE, Chi $\mathrm{JH}$, et al. The effects of thermal preconditioning on oncogenic and intraspinal cord growth features of human glioma cells. Cell Transplant. 2016;25:2099-109.
34. Ropper AE, Zeng X, Haragopal H, Anderson JE, Aljuboori Z, Han I, et al. Targeted treatment of experimental spinal cord glioma with dual gene-engineered human neural stem cells. Neurosurgery. 2016;79:481-91.

35. Ogita S, Endo T, Sugiyama S, Saito R, Inoue T, Sumiyoshi A, et al. Convection-enhanced delivery of a hydrophilic nitrosourea ameliorates deficits and suppresses tumor growth in experimental spinal cord glioma models. Acta Neurochir. 2017;159:939-46.

36. Zhuang Y, Zhao W, Zhang W, Wei H, Huang X, Cai G, et al. A reproducible model of intramedullary spinal cord tumor in rats bearing RG2 cells. Oncotarget. 2017;8:30971-7.

37. Patrizii M, Bartucci M, Pine SR, Sabaawy HE. Utility of glioblastoma patient-derived orthotopic xenografts in drug discovery and personalized therapy. Front Oncol. 2018;8:23. https://doi.org/ 10.3389/fonc.2018.00023.

38. Hidalgo M, Amant F, Biankin AV, Budinská E, Byrne AT, Caldas $\mathrm{C}$, et al. Patient derived xenograft models: an emerging platform for translational cancer research. Cancer Discov. 2014;4:9981013.

39. Li JH, Fan WS, Wang MM, Wang YH, Ren ZG. Effects of mesenchymal stem cells on solid tumor metastasis in experimental cancer models: a systematic review and meta-analysis. J. Transl Med. 2018;16:113. https://doi.org/10.1186/s12967-018-1484-9.

40. Huszthy PC, Daphu I, Niclou SP, Stieber D, Nigro JM, Sakariassen $\mathrm{P} \emptyset$, et al. In vivo models of primary brain tumors: pitfalls and perspectives. Neuro-Oncology. (2012);14:979-993. 